\title{
PERIOPERATIVE DEXMEDETOMIDINE REDUCES DELIRIUM IN ELDERLY PATIENTS AFTER LUNG CANCER SURGERY Ting Huyan ${ }^{1}$, Xuejiao Hu${ }^{2}$, Hourong Peng ${ }^{1}$, Zheng $\mathrm{Zhu}^{3}$, Qi Li ${ }^{1}$ \& Wei Zhang ${ }^{4}$ \\ ${ }^{1}$ Key Laboratory for Space Biosciences and Biotechnology, Institute of Special Environment Biophysics, School of Life Sciences, Northwestern Polytechnical University, Xi'an, China \\ ${ }^{2}$ Department of Laboratory Medicine, West China Hospital, Sichuan University, Chengdu, China \\ ${ }^{3}$ Department of Urology, Xijing Hospital, Fourth Military Medical University, Xi'an, China \\ ${ }^{4}$ Department of Anesthesiology, Henan Provincial People's Hospital \\ (People's Hospital of Zhengzhou University), Zhengzhou, China
}

received: 20.9.2018;

revised: 14.11.2018;

accepted: 22.11 .2018

\section{SUMMARY}

Background: Delirium, which is one of the most disturbing postoperative complications in elderly patients, shows high morbidity in patients undergoing lung cancer surgery. Dexmedetomidine (DEX) is considered a potential prophylactic agent for preventing patients' delirium after lung cancer surgery.

Subjects and methods: Medical records of lung cancer patients over 65 years old with radical pulmonary resection at Henan Provincial People's Hospital from January 2015 to December 2017, China, were evaluated. Patients, care-providers, and investigators were all blinded to group assignment. DEX was administered in the preoperative and intraoperative periods. The incidence of delirium was calculated based on the Intensive Care Delirium Screening Checklist (ICDSC). Scores of $\geq 4$ and 1-3 points represent the diagnoses of delirium and a pre-delirious state, respectively.

Results: During postoperative day 1(POD 1) to POD 7, delirium occurs in both groups. During postoperative POD 1 to POD 7 , the incidence of delirium is lower in the DEX group than that in the control group. Furthermore, there are more mild delirium patients but fewer moderate and severe delirium patients in the DEX group compared with the control group. Finally, patients in the DEX group have a shorter duration of delirium, lower numeric pain rating scale during movement and better sleep quality.

Conclusion: Preoperative and intraoperative application of DEX can reduce the incidence and intensity of delirium after pulmonary resection in elderly patients with lung cancer.

Key words: Dexmedetomidine - delirium - elderly patients - lung cancer surgery

\section{INTRODUCTION}

Thoracic surgery is a vital step in the treatment of lung cancer patients. However, major postoperative complications, especially psychiatric complications, are common after lung cancer surgery (Fernandez et al. 2018, Shiono et al. 2013, Alicia et al. 2017). Delirium, which is defined as an acute syndrome of severe confusion and rapid changes in brain function, has been associated with psychotic symptoms, including inattention, varying degrees of consciousness, hallucinations and delusions (Irwin et al. 2013). Postoperative delirium has been proposed as a risk factor for advancing age, cognitive impairment, white matter microstructural changes, frailty and comorbid medical conditions (Murakawa et al. 2015). In recent years, elderly surgical patients pose a cumulative risk of a high postoperative delirium rate of 15\%-53\% (Florou et al. 2017, Inouye et al. 2014, Shiono \& Abiko 2013). However, strategies that can effectively prevent the occurrence of postoperative delirium are limited (Moyce et al. 2014). Therefore, psychotropic prophylaxis should be addressed to minimize the occurrence of postoperative delirium.

Dexmedetomidine (DEX), a selective $\alpha 2$-adrenergic agonist, has a strong modulating effect on the activity of the sympathetic system (Cai et al. 2014, Sottas \& Anderson 2017, Fan et al. 2017). DEX binds to $\alpha 2-$ receptors present in both the central and peripheral nervous systems. Meanwhile, DEX inhibits the release of norepinephrine and sympathetic activity (Yu et al. 2018). The use of DEX during general anesthesia may reduce postoperative delirium. Thus, DEX currently is increasingly applied as a sedative and as an adjuvant anesthetic during surgery (O'Neil et al. 2014). A previous study showed that DEX was associated with a lower incidence of delirium after lung cancer surgery compared with other sedatives (Liu et al. 2017). However, it is still unclear whether DEX has an antidelirium effect on elderly lung cancer patients.

This study aimed to evaluate the efficacy of using DEX for the prevention of delirium in elderly lung cancer patients who underwent thoracic surgery. Our study was organized as follows: first, the incidence of delirium which was defined as the primary outcome was analyzed and discussed; and second, additional characteristics of delirium were analyzed; third, other main clinical outcomes were observed and discussed. In the end, the conclusions were drawn. This work may provide new prophylaxis strategy to prevent delirium in elderly patients after lung cancer surgery. 


\section{SUBJECTS AND METHODS}

\section{Participants}

The present study included consecutive patients with lung cancer surgery at Henan Provincial People's Hospital from January 2015 to December 2017. The inclusion criteria were patients aged 65 years or older who underwent radical pulmonary resection with body mass index (18 to $25 \mathrm{~kg} / \mathrm{m}^{2}$ ), forced vital capacity (over $80 \%$ ), the first second of forced expiration (over 70\%), American Society of Anesthesiologists (ASA) grade II/III, and no history of metabolic disorder, such as diabetes and blood disorders. Patients were excluded if they met any of the following criteria: patients who were discharged to ICU after surgery; preoperative intensive care delirium screening checklist (ICDSC) (scores over 0); intraoperative new atrial fibrillation; cardiac arrest; stock blood infusion; or hypoxemia ( $\mathrm{SpO}_{2}$ below $90 \%$ over 1 minute).

A total of 412 potential participants were selected, and 346 enrolled patients' data were obtained. During the study period, these consecutively recruited patients were randomly assigned (in a 1:1 ratio) to receive either DEX or placebo (normal saline). This prospective study was approved by the Ethics Committee of Henan Provincial People's Hospital, and written informed consent was obtained from the patients. The study protocol complied with the 1975 Declaration of Helsinki.

\section{Procedures}

The study drugs (DEX or normal saline) were diluted with normal saline to $50 \mathrm{~mL}$ (concentration of DEX was $4 \mu \mathrm{g} / \mathrm{mL})$ before administration. DEX $(0.5 \mu \mathrm{g} / \mathrm{kg})$ was administered in $20 \mathrm{~min}$ before the beginning of the operation followed by a continuous intravenous infusion at a rate of $0.1 \mu \mathrm{g} / \mathrm{kg} / \mathrm{h}$ during the intraoperative period. The drug infusion was stopped $30 \mathrm{~min}$ before the end of the operation.

Etomidate, fentanyl, and rocuronium were intravenously administered for anesthesia induction. A double lumen endotracheal tube was successfully inserted, and the location was confirmed with a fiber bronchoscope. Mechanical ventilation (volume-controlled ventilation) was performed after tracheal intubation with a tidal volume of $8 \mathrm{ml} / \mathrm{kg}$ predicted body weight (PBW) during two-lung ventilation (TLV) and $6 \mathrm{ml} / \mathrm{kg}$ PBW during OLV. The inspired oxygen fraction $\left(\mathrm{F}_{\mathrm{I}} \mathrm{O}_{2}\right)$ was set to 0.5 during TLV and 1.0 during OLV. The respiratory rate was set to 12 breaths/min and adjusted to maintain $\mathrm{P}_{\mathrm{ET}} \mathrm{CO}_{2}$ between 35 and $45 \mathrm{mmHg}$. The positive endexpiratory pressure was maintained at $5 \mathrm{cmH}_{2} \mathrm{O}$. Anesthesia was maintained with propofol, remifentanyl, and cisatracurium. The depth of anesthesia was maintained between BIS 40 and 60 (Aspect, USA) by changing the infusion rate of propofol.

The heart rate (HR), mean arterial pressure, $\mathrm{SpO}_{2}$, and arterial oxygen saturation were also routinely monitored. Bradycardia was defined as $\mathrm{HR}<55$ beats/min; hypotension was defined as $\mathrm{MAP}<55 \mathrm{mmHg}$. Atropine $(0.5 \mathrm{mg})$ or methoxamine (1-2 mg) were administered when bradycardia or hypotension occurred, respectively.

\section{Outcomes and measurements}

Primary outcome: In this study, the primary outcome was the incidence of delirium, assessed daily with the Intensive Care Delirium Screening Checklist (ICDSC) (Bergeron et al. 2001) during the first 7 postoperative days. ICDSC scores of $\geq 4$ were classified as delirium.

Secondary outcomes: The ICDSC score was used to evaluate not only the incidence but also the severity of postoperative delirium. We defined delirium as mild delirium (ICDSC score 4-5), moderate delirium (ICDSC score 6-7) and severe delirium (ICDSC score 8). The data associated with postoperative delirium were collected from postoperative day 1(POD 1) to POD 7. A pre-delirious state (ICDSC scores 1-3 points) was also evaluated. Additionally, other secondary postoperative outcomes, such as initial time of delirium, duration of delirium, numeric pain rating scale (NRS) at rest, NRS during movement, sleep quality (Ritmala-Castren et al. 2014), the overall incidence of non-delirium complications, and length of stay in the hospital after surgery were included in the analysis.

\section{Statistical analysis}

The primary objective of the present study was to investigate the effect of DEX on the incidence of delirium after lung cancer surgery. Based on a previous experiment (Aakerlund \& Rosenberg, 1994), the incidence has been reported to be $21 \%$ in patients undergoing thoracotomy for pulmonary malignancy throughout their hospital stay. To test the hypothesis of this study, sample size was calculated with "Power and Sample Size.com", an online power and sample size calculator, using a power of 0.8 , a $50 \%$ reduction in the primary outcome in the treatment group relative to the control group, and an alpha-error of 0.05 . A sample size of 163 patients in each arm was calculated; taking into account a loss-to-follow-up rate of approximately $7 \%$, we enrolled 173 patients in each group.

The results were presented as the mean \pm standard deviation or percentage or medians. The experimental results were analyzed using SPSS 17.0 software (SPSS, Inc., Chicago, IL, USA). The nominal categorical data between study groups were compared using the chisquared test or Fisher's exact test as appropriate. Oneway ANOVA was used to evaluate the changes in the variables during the study period. The difference (and 95\% CI for the difference) between two medians was calculated with the Hodges-Lehmann estimator. Time to event results were calculated with the Kaplan-Meier estimator, with differences between groups assessed by the log-rank test. A p value of less than 0.05 was considered statistically significant. 


\section{RESULTS}

In total, 412 patients met the recruitment criteria, but 52 patients were excluded due to age, surgery style, or other reasons. Therefore, a total of 360 patients were enrolled and randomly assigned into the DEX group and control group with 180 patients in each group. Seven patients were excluded in each arm. The final analyses included 173 patients in each group (Figure 1). The baseline characteristics of the 346 patients in the study were not different across groups and were shown in Table 1.

The primary outcome of the present study was the effect of DEX on postoperative delirium. During POD 1 to POD 5, our results showed that delirium occurred in both groups (Figure 2A). From POD 1 to POD 5, the incidence of delirium was significantly decreased in the DEX group compared with the control group. In POD 6 and POD 7, no delirium was found in either of the two groups.

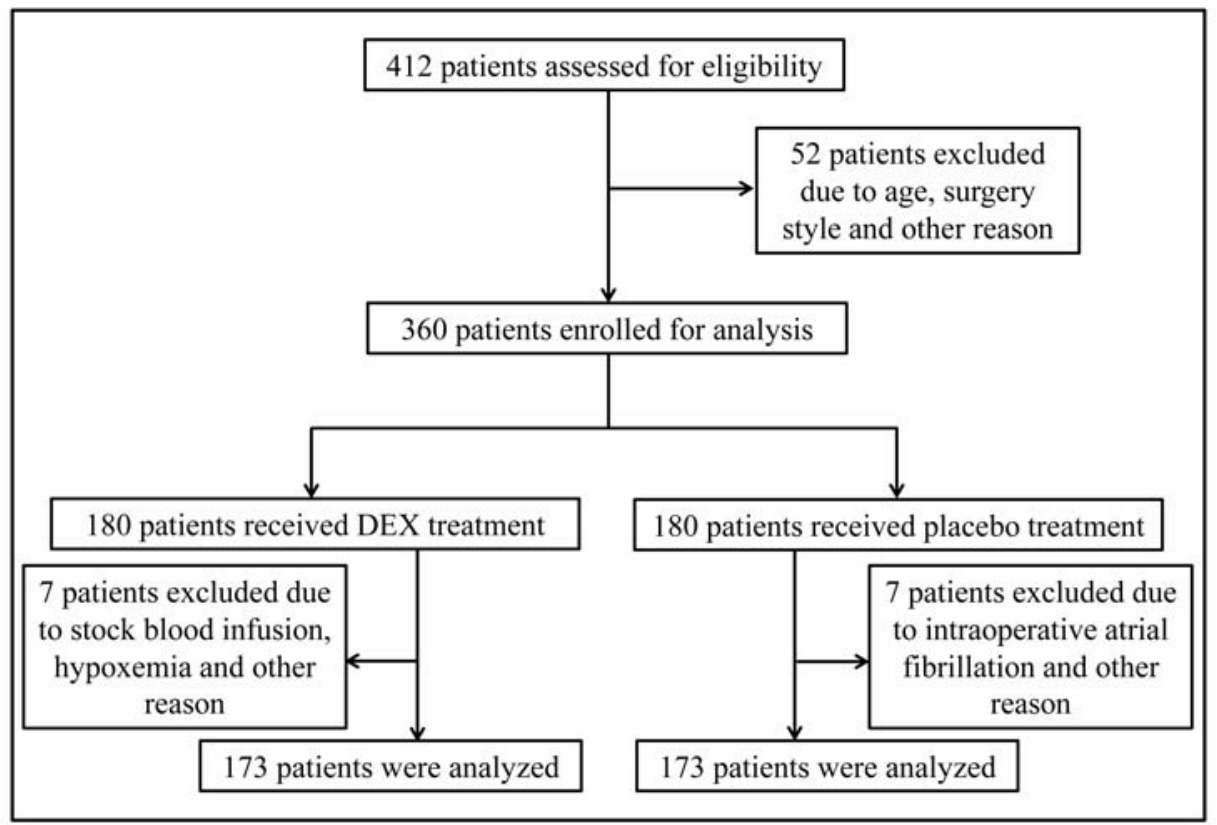

Figure 1. Study flow chart

Table 1. Characteristics of the patients

\begin{tabular}{lcc}
\hline Categories & DEX Group $(\mathrm{n}=173)$ & Control group $(\mathrm{n}=173)$ \\
\hline Age & $70 \pm 5$ & $71 \pm 6$ \\
Sex (male/female) & $89 / 84$ & $94 / 79$ \\
Height (cm) & $168 \pm 11$ & $161 \pm 14$ \\
BMI(kg/m ${ }^{2}$ ) & $22.4 \pm 2.1$ & $24.1 \pm 1.9$ \\
Surgical site (Left/Right) & $100 / 73$ & $98 / 75$ \\
Surgical procedures & & 22 \\
$\quad$ Segmentectomy & 18 & 89 \\
$\quad$ Lobectomy & 85 & 62 \\
$\quad$ Pneumonectomy & 70 & $108 / 65$ \\
ASA (II/III) & $110 / 63$ & $84 \pm 18$ \\
OLV duration (min) & $88 \pm 21$ & $118 \pm 26$ \\
Surgery duration (min) & $124 \pm 32$ & $847 \pm 102$ \\
Infused crystalloid (ml) & $865 \pm 112$ & $146 \pm 29$ \\
Estimated blood loss (ml) & $132 \pm 28$ & $130 \pm 22$ \\
Urinary output (ml) & $144 \pm 31$ & $16 \pm 6$ \\
Awake time in PACU (min) & $18 \pm 5$ & $20 \pm 7$ \\
Extubation time in PACU (min) & $23 \pm 8$ & $13(7.5 \%)$ \\
Preoperative Hemodynamics & & 10 \\
$\quad$ Bradycardia (n, \%) & $18(10.4 \%)$ & $9(5.2 \%)$ \\
$\quad$ Atropine & 15 & 6 \\
$\quad$ Hypotension (n, \%) & $12(6.9 \%)$ & 8 \\
\hline
\end{tabular}


A

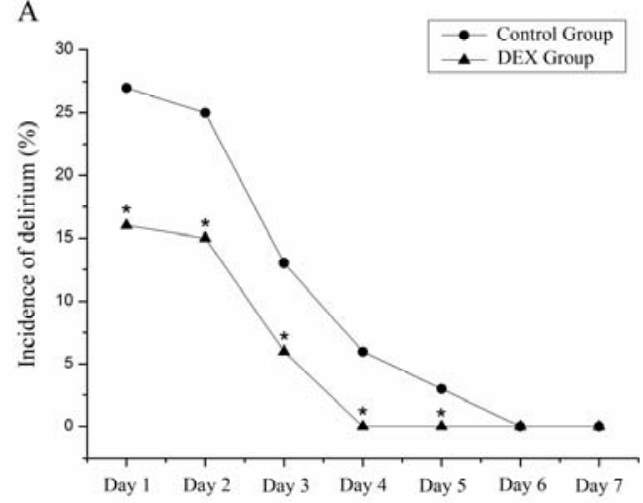

C

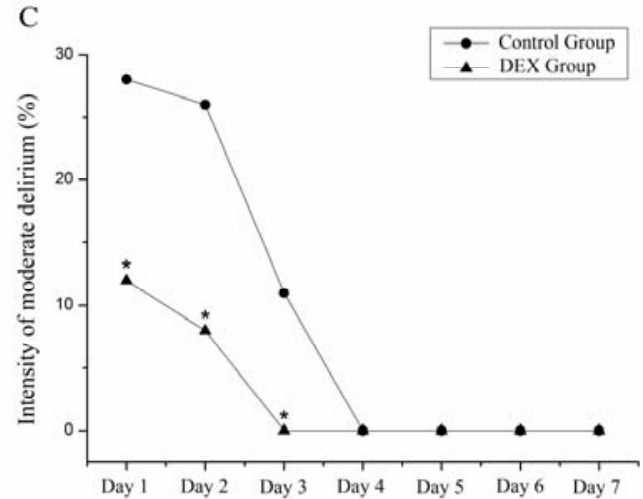

B

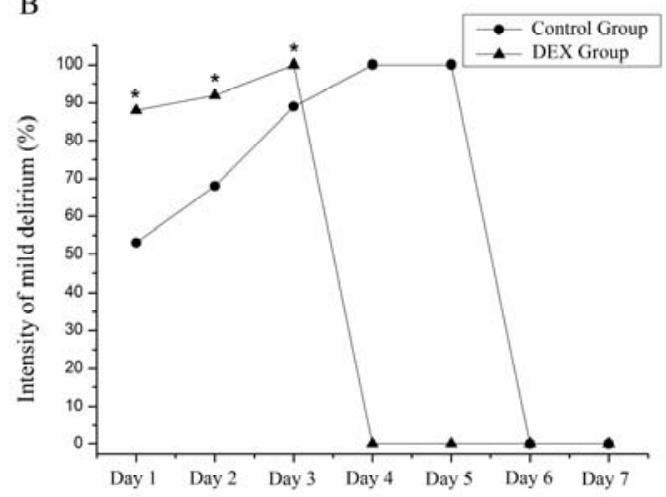

D

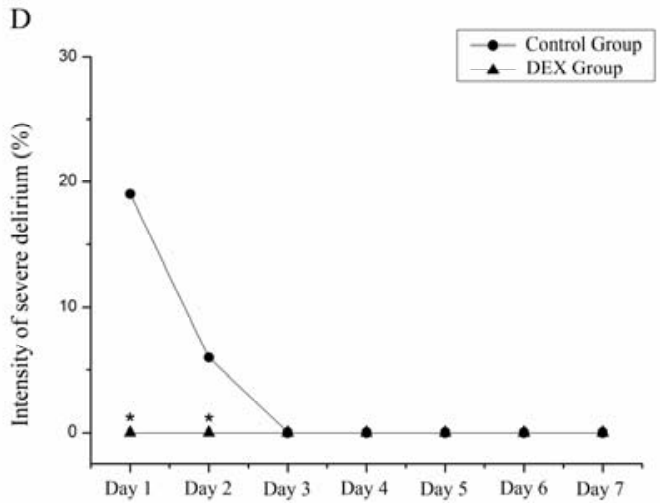

Figure 2. Daily incidence and intensity of postoperative delirium

Table 2. Secondary outcomes of postoperative delirium patients after DEX treatment

\begin{tabular}{lcc}
\hline Categories & DEX Group (n=173) & Control group (n=173) \\
\hline Time to onset of delirium after surgery(days) & $2(1$ to 3$)$ & $2(1$ to 3$)$ \\
Duration of delirium(days) & $2(1 \text { to } 3)^{*}$ & $4(3$ to 5$)$ \\
Pre-delirious state & & $22(12.7 \%)$ \\
$\quad$ IDSC score=3 (n, \%) & $12(6.9 \%)^{*}$ & $6(3.5 \%)$ \\
IDSC score=2 (n, \%) & $8(4.6 \%)$ & $6(3.5 \%)$ \\
IDSC score=1 (n, \%) & $5(2.9 \%)$ & $3(1$ to 4$)$ \\
NRS for patients at rest (score) & & $3(1$ to 4$)$ \\
1h after surgery & $2(1$ to 4$)$ & $4(2$ to 5$)$ \\
6h after surgery & $2(1$ to 3$)$ & $6(4$ to 7$)$ \\
24h after surgery & $3(1$ to 4$)$ & $4(3$ to 5$)$ \\
NRS for patients at movement (score) & & $5(3$ to 6$)$ \\
1h after surgery & $3(1 \text { to } 4)^{*}$ & $4(2$ to 5$)$ \\
6h after surgery & $4(2$ to 5$)$ & $5(4$ to 6$)$ \\
24h after surgery & & $4(3$ to 5$)$ \\
Sleep quality(score) & $3(2$ to 4$)$ \\
The first morning after surgery & $2(1 \text { to } 4)^{*}$ & $16(12.3 \%)$ \\
The second morning after surgery & $2(0$ to 3$)$ & $9(7$ to 11$)$ \\
Third morning after surgery & $3(2$ to 4$)$ & $18(11.7 \%)$ \\
Overall incidence of non-delirium complications (n, \%) & $8(6$ to 10$)$ & \\
Length of stay in the hospital after surgery & &
\end{tabular}

$$
{ }^{*} \mathrm{P}<0.05
$$

A: Daily incidence of postoperative delirium in the DEX group and control group; B: Daily intensity of mild delirium in the DEX group and control group; C: Daily intensity of moderate delirium in the DEX group and control group; D: Daily intensity of severe delirium in the DEX group and control group.
Secondary outcomes are shown in Figure 2 (B, C, D) and Table 2 . The intensity of delirium was also analyzed and shown in Figure $2(\mathrm{~B}, \mathrm{C}, \mathrm{D})$. At POD 1, the intensity of delirium (mild, moderate and severe) was $88 \%, 12 \%$ and 0 in the DEX group and $53 \%, 28 \%$ and $19 \%$ in the control group respectively. In POD 2, the 
intensity of delirium (mild, moderate and severe) was $92 \%, 8 \%$ and $0 \%$ in DEX group and $68 \%, 26 \%$ and $6 \%$ in control group respectively. In POD 3, the intensity of delirium (mild, moderate and severe) was $100 \%, 0 \%$ and $0 \%$ in DEX group and $89 \%, 11 \%$ and $0 \%$ in the control group respectively. From POD 4 to POD 5, only the low intensity of delirium was found in the control group. From POD 1 to POD 3, the intensity of mild and moderate in the DEX group were higher and lower, respectively, than in the control group, and the intensity of severe was higher in the control group than in the DEX group.

The duration of delirium was shorter in the DEX group than that in the control group. The percentage of patients with an ICDSC score 3 in the DEX group was decreased compared with the number in the control group. NRS during movement was lower in the DEX group than that in the control group only at $1 \mathrm{~h}$ after surgery. The sleep quality assessed the first morning after surgery was higher in the DEX group than that in the control group (Table 2).

\section{DISCUSSION}

In recent years, several reports have shown that the number of older adults over age 65 in China rapidly approached 1.5 billion, equaling an unprecedented $10.8 \%$ of the population, with the prediction reaching $17.17 \%$ by 2020 (Stewart Williams et al. 2017). Postoperative delirium in elderly people is an acute disorder of attention and cognition that is common, fatal, costly, and underrecognized. Accordingly, delirium is now included on elderly patients' safety agendas and has been increasingly used as an indicator of health-care quality for elderly people.

The main observational index in this study was the incidence of postoperative delirium. The postoperative observation time window was from POD 1 to POD 7, and the results showed that delirium occurred in both groups. The incidence of delirium in the DEX group from POD 1 to POD 5 was significantly lower than in the control group, and there was no difference between the two groups at POD 6 and POD 7 postoperatively. Previous studies have shown that postoperative delirium occurred mostly on POD 1-3. The duration of delirium in the DEX group was shorter than in the control group. These data indicate that DEX can decrease the duration of postoperative delirium; that is, the application of DEX can accelerate the recovery time. Additionally, postoperative delirium usually occurs within the first few days after surgery peaks between 1 and 3 days postoperatively (Whitlock et al. 2011). Su et al. suggested that a low dose of DEX could effectively decrease the occurrence of delirium ( $\mathrm{Su}$ et al. 2016). Thus, the effects of DEX on some other features of delirium, such as the initial time of delirium and the duration of delirium, were evaluated in our study. Moreover, postoperative ICDSC scores of 1-3 were defined as a pre-delirious state. The higher the score is the closer the patient is to the delirium state and the greater the interference to the body. We carried out statistics on the incidence of different scores. The results showed that DEX could reduce the incidence of ICDSC scores of 3 points, but there was no difference in the incidence of ICDSC scores of 1 point and 2 points.

The prognosis and clinical treatment of patients are related to the degree of delirium experienced. For this reason, further analysis of the degree of delirium was carried out. Delirium in our present study was classified as mild, moderate and severe according to the ICDSC score. The incidence of mild delirium was higher in the DEX group than that in the control group on POD 1 to POD 3, whereas the incidence of moderate delirium was higher in the control group than in the DEX group on POD 1 to POD 3. Severe delirium was found in the control group on POD 1 and POD 2. However, no severe delirium was found in the DEX group. From POD 4 to POD 5, the degree of delirium in the control group was only mild, but no delirium was present in the DEX group. This indicates that DEX can reduce the incidence of postoperative delirium. Moreover, the severity of postoperative delirium was significantly lower in elderly patients after thoracic surgery.

The occurrence of postoperative delirium is associated with worse outcomes including prolonged mechanical ventilation, increased postoperative complications, high mortality rate and long-term hospital stays ( $\mathrm{Su}$ et al. 2016). In the present study, we mainly examined variable relevant to postoperative delirium, such as pain, sleep quality, the overall incidence of non-delirium complications, and the length of stay in the hospital after surgery.

Sleep disturbances are common in postoperative patients, and poor sleep is associated with a higher prevalence of postoperative delirium (Flink et al. 2012). Studies have reported that the quality of sleep at the night after surgery has been associated with postoperative delirium (Shigeta et al. 2001, Yildizeli et al. 2005). Patients with better sleep quality may have a lower incidence of delirium. Meanwhile, DEX is considered to have a sleep-promoting effect (Nelson at al. 2003). The results of this study showed that although there was no difference in sleep quality between the two groups on POD 2 and POD 3 after surgery, DEX significantly improved the quality of sleep on the first night after surgery, which contributes to postoperative recovery.

Pain is a risk factor for the development of delirium (Kosar et al. 2014), and postoperative pain has been considered an important precipitating factor, and the level of postoperative pain is related to delirium (Leung et al. 2013). DEX can provide analgesia by acting on $\alpha 2$ adrenergic receptors in the spinal cord (Lin et al. 2009). Higher levels of analgesia can help reduce postoperative delirium. The worse the level of analgesia, the more susceptible the patient is to suffering emotional disturbances or even delirium. This study evaluated the analgesic level at $1 \mathrm{~h}, 6 \mathrm{~h}$, and $24 \mathrm{~h}$ after surgery, including pain at rest and movement. Compared with the control 
group, the DEX group only experienced reduced pain during movement $1 \mathrm{~h}$ after surgery, which may favor decreasing the incidence of delirium. Although DEX has been shown to significantly improve short-term and long-term outcomes in patients undergoing non-cardiac surgery after ICU admission (Su et al. 2016), our study showed no difference between postoperative non-delirium complications and length of stay in the hospital.

There are also some limitations to this study. First, delirium diagnosis are based on one or a combination of clinical indexes, such as clinical notes, physical examination, cognitive testing, and laboratory results (Neufeld et al. 2014). In this study, the diagnosis was mainly based on one test, and a more objective and reliable screening tool may improve the detection of delirium (Numan et al. 2017). Furthermore, reasonable electroencephalography monitoring could reduce the incidence of postoperative delirium (Aldecoa et al. 2017, Siddiqi et al. 2016). In this study, only the bispectral index (from 40 to 60) was used for monitoring the depth of anesthesia.

\section{CONCLUSION}

In conclusion, this study indicates that in elderly patients after lung resection, preoperative and intraoperative application of DEX can decrease the incidence of delirium. It can also decrease the intensity of delirium and shorten the duration of delirium. Furthermore, DEX may improve postoperative outcomes such as less pain and better sleep quality. More studies are warranted to confirm these results.

\section{Acknowledgements:}

This study was supported by the grants from Henan Provincial Health System Training Program for Study Abroad (2017060), Science and Technology Project Henan Province (182102310167), Medical Science Research Project of Henan Province (201602227), the National Natural Science Foundation of China (NSFC) (31500688 and 81502465), and the Fundamental Research Funds for the Central Universities (3102016OQD042).

\section{Conflict of interest: None to declare.}

\section{Contribution of individual authors:}

All authors contributed equally to this manuscript.

\section{References}

1. Aakerlund LP, Rosenberg J: Postoperative delirium: treatment with supplementary oxygen. Br J Anaesth 1994; 72:286-290

2. Aldecoa C, Bettelli G, Bilotta F, Sanders RD, Audisio R, Borozdina A, et al.: European Society of Anaesthesiology evidence-based and consensus-based guideline on postoperative delirium. Eur J Anaesthesiol 2017; 34:192-214
3. Alicia M, Dario L: Analysis of the effect of a psychological intervention program group on quality of life of oncology patients. Rev Argent Clin Psic 2017; 2:202-209

4. Bergeron N, Dubois MJ, Dumont M, Dial S, Skrobik Y: Intensive Care Delirium Screening Checklist: evaluation of a new screening tool. Intensive Care Med 2001; 27:859-864

5. Cai Y, Xu H, Yan J, Zhang L, Lu Y: Molecular targets and mechanism of action of dexmedetomidine in treatment of ischemia/reperfusion injury. Mol Med Rep 2014; 9:1542-1550

6. Cavaliere F, D'Ambrosio F, Volpe C, Masieri $S$ : Postoperative delirium. Curr Drug Targets 2005; 6:807814

7. Fan H, Zhao Y, Sun M, Ye JH, Chen GD, Zhu JH: Dexmedetomidine based sedation for post-surgery critically ill adults: a meta-analysis of randomized controlled trials. Iran J Public Health 2017;46:1611-1622

8. Fernandez FG, Kosinski AS, Furnary AP, Onaitis M, Kim $S$, Habib RH, et al.: Differential effects of operative complications on survival after surgery for primary lung cancer. J Thorac Cardiovasc Surg 2018; 155:1254-1264

9. Flink BJ, Rivelli SK, Cox EA, White WD, Falcone G, Vail TP, et al.: Obstructive sleep apnea and incidence of postoperative delirium after elective knee replacement in the nondemented elderly. Anesthesiology 2012; 116:788796

10. Florou C, Theofilopoulos D, Tziaferi S, Chania M: PostOperative delirium in elderly people diagnostic and management issues of post-operative delirium in elderly people. Adv Exp Med Biol 2017; 987:301-312

11. Inouye SK, Westendorp RG, Saczynski JS: Delirium in elderly people. Lancet 2014; 383:911-922

12. Irwin SA, Pirrello RD, Hirst JM, Buckholz GT, Ferris FD: Clarifying delirium management: practical, evidencedbased, expert recommendations for clinical practice. $J$ Palliat Med 2013; 16:423-435

13. Kosar CM, Tabloski PA, Travison TG, Jones RN, Schmitt EM, Puelle MR: Effect of preoperative pain and depressive symptoms on the development of postoperative delirium. Lancet Psychiatry 2014; 1:431-436

14. Leung JM, Sands LP, Lim E, Tsai TL, Kinjo S: Does preoperative risk for delirium moderate the effects of postoperative pain and opiate use on postoperative delirium? Am J Geriatr Psychiatry 2013; 21:946-956

15. Lin TF, Yeh YC, Lin FS, Wang YP, Lin CJ, Sun WZ, et al.: Effect of combining dexmedetomidine and morphine for intravenous patient-controlled analgesia. $\mathrm{Br} J$ Anaesth 2009; 102:117-122

16. Liu X, Xie G, Zhang $K$, Song S, Song F, Jin Y, et al.: Dexmedetomidine vs propofol sedation reduces delirium in patients after cardiac surgery: A meta-analysis with trial sequential analysis of randomized controlled trials. $J$ Crit Care 2017; 38:190-196

17. Moyce Z, Rodseth RN, Biccard BM: The efficacy of perioperative interventions to decrease postoperative delirium in non-cardiac surgery: a systematic review and metaanalysis. Anaesthesia 2014; 69:259-269

18. Murakawa K, Kitamura Y, Watanabe S, Hongo S, Shinomiya $K$, Sendo T: Clinical risk factors associated with postoperative delirium and evaluation of delirium management and assessment team in lung and esophageal cancer patients. J Pharm Health Care Sci 2015; 1:4 
19. Nelson LE, Lu J, Guo T, Saper CB, Franks NP, Maze M: The alpha2-adrenoceptor agonist dexmedetomidine converges on an endogenous sleep-promoting pathway to exert its sedative effects. Anesthesiology 2003; 98:428-436

20. Neufeld KJ, Nelliot A, Inouye SK, Ely EW, Bienvenu OJ, Lee HB, et al.: Delirium diagnosis methodology used in research: a survey-based study. Am J Geriatr Psychiatry 2014; $22: 1513-1521$

21. Numan T, van den Boogaard M, Kamper AM, Rood PJT, Peelen LM, Slooter AJC, et al.: Recognition of Delirium in Postoperative Elderly Patients: A Multicenter Study. J Am Geriatr Soc 2017; 65:1932-1938

22. O'Neil T, Rodgers PE, Shultz C: Dexmedetomidine as adjuvant therapy for acute postoperative neuropathic pain crisis. J Palliat Med 2014; 17:1164-1166

23. Ritmala-Castren M, Lakanmaa RL, Virtanen I, Leino-Kilpi $H$ : Evaluating adult patients' sleep: an integrative literature review in critical care. Scand $J$ Caring Sci 2014; 28:435-448

24. Shigeta H, Yasui A, Nimura Y, Machida N, Kageyama M, Miura $M$, et al.: Postoperative delirium and melatonin levels in elderly patients. Am J Surg 2001; 182:449-454

25. Shiono S, Abiko M, Sato T: Postoperative complications in elderly patients after lung cancer surgery. Interact Cardiovasc Thorac Surg 2013; 16:819-823
26. Siddiqi N, Harrison JK, Clegg A, Teale EA, Young J, Taylor $J$, et al.: Interventions for preventing delirium in hospitalised non-ICU patients. Cochrane Database Syst Rev 2016; 3:CD005563

27. Sottas CE, Anderson BJ: Dexmedetomidine: the new allin-one drug in paediatric anaesthesia? Curr Opin Anaesthesiol 2017; 30:441-451

28. Stewart Williams J, Norstrom F, Ng N: Disability and ageing in China and India - decomposing the effects of gender and residence. Results from the WHO study on global AGEing and adult health (SAGE). BMC Geriatr 2017; 17:197

29. Su X, Meng ZT, Wu XH, Cui F, Li HL, Wang DX, et al.: Dexmedetomidine for prevention of delirium in elderly patients after non-cardiac surgery: a randomised, double-blind, placebo-controlled trial. Lancet 2016; 388:1893-1902

30. Whitlock EL, Vannucci A, Avidan MS: Postoperative delirium. Minerva Anestesiol 2011; 77:448-56

31. Yildizeli B, Ozyurtkan MO, Batirel HF, Kuscu K, Bekiroglu N, Yuksel M: Factors associated with postoperative delirium after thoracic surgery. Ann Thorac Surg 2005; 79:1004-1009

32. Yu X, Franks NP, Wisden W: Sleep and Sedative States Induced by Targeting the Histamine and Noradrenergic Systems. Front Neural Circuits 2018; 12:4

\section{Correspondence:}

Wei Zhang, MD

Department of Anesthesiology, Henan Provincial People's Hospital

(People's Hospital of Zhengzhou University)

Zhengzhou, China

E-mail:myhope2005@163.com 